\title{
ON THE TOTAL VARIATION OF SOLUTIONS OF THE BOUNDED VARIATION MOMENT PROBLEM ${ }^{1}$
}

\author{
MELVIN BLOOM
}

1. Introduction. In this paper we consider functions $\phi(t)$ which satisfy for a given real sequence $\left\{\mu_{n}\right\}$ the equations

$$
\mu_{n}=\int_{a}^{\infty} t^{n} d \phi(t), \quad n=0,1,2, \cdots,
$$

where the integrals converge absolutely. Any such function $\phi(t)$ is called a solution of the moment problem $(\mu, a)$ or simply a solution of $(\mu, a)$.

Boas [1 $]^{2}$ first pointed out that for an arbitrary sequence $\left\{\mu_{n}\right\}$ there exist infinitely many solutions of $(\mu, 0)$; in fact, he showed that any such sequence can be decomposed in infinitely many ways into the difference of two (Stieltjes) sequences $\left\{\lambda_{n}\right\}$ and $\left\{\nu_{n}\right\}$ where both $(\lambda, 0)$ and $(\nu, 0)$ have nondecreasing solutions. In this theorem the choice of $\lambda_{0}$ and $\nu_{0}$ is subject only to the conditions

$$
\lambda_{0}>0, \quad \nu_{0}>0, \quad \lambda_{0}-\nu_{0}=\mu_{0} .
$$

For arbitrary $\epsilon>0$ we can choose $\lambda_{0}=\mu_{0}+\epsilon / 2, \nu_{0}=\epsilon / 2$, or $\lambda_{0}=\epsilon / 2$, $\nu_{0}=-\mu_{0}+\epsilon / 2$, according as $\mu_{0} \geqq 0$ or $\mu_{0}<0$, and it follows that there exists a solution $\phi(t)$ of $(\mu, 0)$ having total variation less than $\left|\mu_{0}\right|+\epsilon$.

If $\psi(t)$ is a solution of $(\lambda, 0), \lambda_{n}=\sum_{0}^{\infty}(-1)^{i} C_{n, i} a^{i} \mu_{n}, a$ arbitrary, such that the total variation of $\psi(t)$ is less than $\left|\lambda_{0}\right|+\epsilon$, then $\phi(t)$ $=\psi(t-a)$ is a solution of $(\mu, a)$ with total variation less than $\left|\mu_{0}\right|+\epsilon$. The proof of the following theorem is now easy.

THEOREM 1.1. Let $\psi(t)$ be a solution of $(\lambda,-\infty)$ and $\left\{\mu_{n}\right\}$ be a sequence with $\mu_{0}=\lambda_{0}$. Then for arbitrary $a$ and $\epsilon>0$ there exists $a \phi(t)$ satisfying

$$
\begin{aligned}
\mu_{n}= & \int_{-\infty}^{a} t^{n} d \psi(t)+\int_{a}^{\infty} t^{n} d(\psi(t)+\phi(t)), \quad n=0,1,2, \cdots, \\
& \int_{-\infty}^{\infty}|d \phi(t)|<\epsilon .
\end{aligned}
$$

Received by the editors April 14, 1952.

1 The material in this paper constitutes a part of a thesis submitted to Northwestern University and prepared under the direction of Professor Walter T. Scott.

2 Numbers in brackets refer to references at the end of this paper. 
Pólya [2] has shown that there are infinitely many entire transcendental solutions of $(\mu,-\infty)$ and [3] that there are infinitely many step function solutions with discontinuities restricted to an arbitrarily preassigned set of points with no finite limit points. In \$2, using the method of Pólya, we show that there exists an entire transcendental solution of $(\mu, 0)$ with total variation on the whole real axis arbitrarily near $\left|\mu_{0}\right|$, and in $\$ 3$, using Pólya's method, we do the same for the step functions. In $\$ 4$ we give a method for constructing such a step function with discontinuities restricted to the points $a$, $a^{2}, a^{3}, \cdots ; a \geqq 2$.

\section{Entire transcendental solutions.}

THEOREM 2.1. For $\epsilon>0$ there exists an entire transcendental solution $\phi(t)$ of $(\mu, 0)$ such that $\int_{-\infty}^{\infty}|d \phi(t)|<\left|\mu_{0}\right|+\epsilon$.

We shall use the following lemma.

LEMMa 2.1. For any positive integer $n$, any real $A^{\prime} \neq 0$, and any $\epsilon^{\prime}>0$, there exists an entire transcendental function $g(z)$ satisfying the following three conditions:

$$
\begin{aligned}
& \int_{0}^{\infty} t^{i} g(t) d t= \begin{cases}0, & j=0,1, \cdots, n-1, \\
A^{\prime}, & j=n,\end{cases} \\
& \int_{-\infty}^{\infty} t^{i}|g(t)| d t<\epsilon^{\prime}, i=0,1, \cdots, n-1, \\
&|g(z)|<\epsilon^{\prime},|z| \leqq n .
\end{aligned}
$$

We remark that this lemma is the same as Polya's [2] except for the interval of integration in (2.1). He uses $e^{-t^{2}}$ as the basis for the construction of the function of his theorem. We now prove the lemma.

For fixed real $\lambda \neq 0,1$ define

$$
f(z)=\frac{(-1)^{n} 2 \lambda}{\pi^{1 / 2} n !(\lambda-1)} \frac{d^{n}}{d z^{n}}\left(e^{-z^{2}}-e^{-\lambda^{2} z^{2}}\right) .
$$

Then $|f(z)|<M<\infty,|z| \leqq 1$,

$$
\begin{array}{ll}
\int_{0}^{\infty}{ }^{i} f(t) d t= \begin{cases}0, & j=0,1, \cdots, n-1, \\
1, & j=n,\end{cases} \\
\int_{-\infty}^{\infty}\left|t^{i}\right||f(t)| d t<L<\infty, & j=0,1, \cdots, n-1 .
\end{array}
$$

Choose $\alpha>0$ so that $\left|A^{\prime}\right| L \alpha<n,\left|A^{\prime}\right| M \alpha^{n+1}<\epsilon^{\prime}$, and $n \alpha<1$. Then 
for $\beta=A^{\prime} \alpha^{n+1}, \beta f(\alpha z)$ satisfies the three conditions of the lemma.

To prove the theorem let $g_{0}(z)=\left[2 \lambda \mu_{0} / \pi^{1 / 2}(\lambda-1)\right]\left(e^{-z^{2}}-e^{-\lambda^{2} z^{2}}\right)$ and for indices $n=1,2,3, \cdots$ use the lemma with $A^{\prime}$ and $\epsilon^{\prime}$ replaced by $A_{n}$ and $2^{-n} \epsilon$ to choose recursively the functions $g_{n}(z)$ satisfying

$$
\int_{0}^{\infty} t^{i} g_{n}(t) d t= \begin{cases}0, & j=0,1, \cdots, n-1, \\ A_{n}, & j=n,\end{cases}
$$

where

$$
A_{n}=\mu_{n}-\int_{0}^{\infty} t^{n}\left(g_{0}(t)+\cdots+g_{n-1}(t)\right) d t .
$$

It is readily verified that any $\phi(t)$ for which $\phi^{\prime}(t)=\sum_{0}^{\infty} g_{i}(t)$ satisfies the conditions of the theorem.

3. Step function solutions. Here we neglect $\mu_{0}$. This is no loss, since a solution for the sequence $\mu_{0}, \mu_{1}, \mu_{2}, \cdots$ becomes a solution for the sequence $\mu_{0}^{\prime}, \mu_{1}, \mu_{2}, \cdots$ by the addition at the origin of a discontinuity with saltus $\left(\mu_{0}^{\prime}-\mu_{0}\right)$.

Theorem 3.1. If the sequence $0<b_{1}<b_{2}<\cdots$ has limit point infinity, then for $\epsilon>0$ and any sequence $\left\{\mu_{n}\right\}$ there exists a solution of the infinite system

$$
\sum_{i=1}^{\infty} b_{i}^{n} u_{i}=\mu_{n}, \quad n=1,2,3, \cdots,
$$

satisfying the following two conditions:

$$
\begin{aligned}
& \sum_{i=1}^{\infty} b_{i}^{n}\left|u_{i}\right|<\infty, \quad n=1,2,3, \cdots, \\
& \sum_{i=1}^{\infty}\left|u_{i}\right|<\epsilon .
\end{aligned}
$$

This theorem is a corollary of

THEOREM 3.2. Let the matrix $A=\left(a_{j k}\right)$ with complex elements satisfy the following three conditions:

$$
\left\{\begin{array}{c}
\text { Every segment of the form } \\
a_{1, q+1}, a_{1, q+2}, \cdots, \\
\cdot . \cdot \cdot \cdot \cdots, \\
a_{n, q+1}, a_{n, q+2}, \cdots \\
\text { is of rank } n,
\end{array} \quad n=2,3,4, \cdots,\right.
$$




$$
\begin{aligned}
\lim _{k \rightarrow \infty} \frac{a_{j-1, k}}{a_{j, k}} & =0, \quad j=2,3,4, \cdots, \\
\limsup _{k \rightarrow \infty}\left|a_{1 k}\right| & =\infty .
\end{aligned}
$$

Then for arbitrary $\epsilon>0$ and arbitrary $\mu=\left(\mu_{1}, \mu_{2}, \mu_{8}, \cdots\right)$ there exists $a u=\left(u_{1}, u_{2}, u_{3}, \cdots\right)$ satisfying the following three conditions:

$$
\begin{aligned}
A u & =\mu, \\
\sum_{k=1}^{\infty}\left|a_{j k} u_{k}\right| & <\infty, \quad j=1,2,3, \cdots, \\
\sum_{k=1}^{\infty}\left|u_{k}\right| & <\epsilon .
\end{aligned}
$$

Condition (3.3) is necessary for condition (3.6).

The following lemma will be used in the proof of the theorem.

LEMMA 3.1. Let the matrix ( $\left.a_{j k}\right)$ satisfy conditions (3.1), (3.2), and (3.3). Then for arbitrary $\epsilon^{\prime}>0$, arbitrary positive integers $n$ and $q$, and arbitrary number $\mu^{\prime}$, there exists an integer $q^{\prime}>q+n$ and numbers $u_{q+1}, u_{q+2}, \cdots, u_{q^{\prime}}$ satisfying the following three conditions:

$$
\begin{aligned}
a_{j, q+1} u_{q+1}+\cdots+a_{j q^{\prime}} u_{q^{\prime}} & = \begin{cases}0, & j=1,2, \cdots, n-1, \\
\mu^{\prime}, & j=n,\end{cases} \\
\left|a_{j, q+1} u_{q+1}\right|+\cdots+\left|a_{j q^{\prime}} u_{q^{\prime}}\right|<\epsilon^{\prime}, & j=1,2, \cdots, n-1, \\
\left|u_{q+1}\right|+\cdots+\left|u_{q^{\prime}}\right|<\epsilon^{\prime} . &
\end{aligned}
$$

We remark that Pólya [3] proves Theorem 3.2 with conditions (3.3) and (3.6) deleted. His proof depends upon a lemma which is Lemma 3.1 with conditions (3.3) and (3.9) deleted.

We now prove the lemma.

Condition (3.1) implies the existence of a set of indices $k_{1}, k_{2}, \cdots$, $k_{n}, q<k_{1}<k_{2}<\cdots<k_{n}$ for which the determinant of the system

$$
a_{j k_{1}} u_{k_{1}}+\cdots+a_{j k_{n}} u_{k_{n}}=x_{j}, \quad j=1,2, \cdots, n,
$$

does not vanish and hence there exists a $\delta>0$ such that

$$
\left|a_{j k_{1}} u_{k_{1}}\right|+\cdots+\left|a_{j k_{n}} u_{k_{n}}\right|<\min \left(\frac{\epsilon^{\prime}}{2}, \frac{M \epsilon^{\prime}}{2 n}\right)
$$

for $\left|x_{j}\right|<\delta, j=1,2, \cdots, n$, where $M$ is the smallest of the absolute values of the nonzero elements in the coefficient matrix of the system (3.10). The number $\delta$ is now fixed. 
Condition (3.3) implies the existence of an infinite sequence of indices $l_{1}<l_{2}<l_{3}<\cdots$ for which $\left|a_{1 l_{i}}\right| \geqq 1$. Condition (3.2) gives the existence of an index $K$ such that for $k>K$,

$$
\frac{\left|\mu^{\prime} a_{j k}\right|}{\left|a_{n k}\right|}<\min \left(\delta, \frac{\epsilon^{\prime}}{2}, \frac{M \epsilon^{\prime}}{2 n}\right), \quad j=1,2, \cdots, n-1 .
$$

Let $q^{\prime}=l_{i}$ for some $l_{i}>\operatorname{Max}\left(K, k_{n}\right)$ so that

$$
\frac{\left|\mu^{\prime}\right|}{\left|a_{n q^{\prime}}\right|}<\frac{\left|\mu^{\prime} a_{j q^{\prime}}\right|}{\left|a_{n q^{\prime}}\right|}, \quad j=1,2, \cdots, n-1 .
$$

With $\delta$ and $q^{\prime}$ now fixed put $x_{n}=0$ and

$$
\begin{aligned}
& x_{j}=-\frac{\mu^{\prime} a_{j q^{\prime}}}{a_{n q^{\prime}}}, \quad j=1,2, \cdots, n-1, \\
& u_{q^{\prime}}=\frac{\mu^{\prime}}{a_{n q^{\prime}}} \\
& u_{k}=0, \quad q<k<q^{\prime}, k \neq k_{1}, k_{2}, \cdots, k_{n} .
\end{aligned}
$$

It is readily verified that the numbers $u_{q+1}, u_{q+2}, \cdots, u_{q^{\prime}}$ so determined satisfy conditions (3.7), (3.8), and (3.9).

We turn to the proof of the theorem and consider first the necessity of condition (3.3) for condition (3.6). If lim $\sup _{k \rightarrow \infty}\left|a_{1 k}\right|<\infty$ the sequence $\left\{\left|a_{1 k}\right|\right\}$ has an upper bound $B$. The sequence $\left\{\mu_{i}\right\}$ is arbitrary and, for $\mu_{1} \neq 0$, there exists an $n$ for which $\left|\sum_{1}^{n} a_{1 k} u_{k}-\mu_{1}\right|<\left|\mu_{1}\right| / 2$. Then $\sum_{k=1}^{n}\left|u_{k}\right|>\left|\mu_{1}\right| / 2 B$ and condition (3.6) cannot be satisfied.

To complete the proof of the theorem we note that condition (3.3) implies the existence of an index $q_{1}$ for which $\left|a_{1 q_{1}}\right|>2\left|\mu_{1}\right| / \epsilon$. Let

$$
u_{k}= \begin{cases}0, & k=1,2, \cdots, q_{1}-1, \\ \mu_{1} / a_{1 q_{1}}, & k=q_{1} .\end{cases}
$$

Then

$$
\sum_{k=1}^{q_{1}} a_{j k} u_{k}=\mu_{1}
$$

and

$$
\sum_{k=1}^{q_{1}}\left|u_{k}\right|<\epsilon / 2
$$

This completes the first step.

At the $n$th step, $n>1$, we let $\mu^{\prime}, \epsilon^{\prime}, n, q$, and $q^{\prime}$ of the lemma be 
$\mu_{n}-\sum_{j=1}^{\ell_{n}-1} a_{n j} u_{j}, 2^{-n} \epsilon, n, q_{n-1}$, and $q_{n}$ respectively and we have

$$
\begin{aligned}
& \sum_{k=q_{n-1}+1}^{q_{n}} a_{j k} u_{k}= \begin{cases}0, & j=1,2, \cdots, n-1, \\
\mu_{n}, & j=n,\end{cases} \\
& \sum_{k=q_{n-1}+1}^{q_{n}}\left|a_{j k} u_{k}\right|<2^{-n_{\epsilon}}, \quad j=1,2, \cdots, n-1, \\
& \sum_{k=q_{n-1}+1}^{q_{n}}\left|u_{k}\right|<2^{-n^{-}} \epsilon .
\end{aligned}
$$

Combining the results of the first $n$ steps we have

$$
\begin{array}{lr}
\sum_{k=1}^{q_{n}} a_{j k} u_{k}=\mu_{j}, & j=1,2, \cdots, n, \\
\sum_{k=1}^{q_{n}}\left|a_{j k} u_{k}\right|<\sum_{k=1}^{q_{j}}\left|a_{j k} u_{k}\right|+\epsilon, & j<n, \\
\sum_{k=1}^{q_{n}}\left|u_{k}\right|<\epsilon . &
\end{array}
$$

These conditions hold for all $n$, and this completes the proof of the theorem.

We now prove a theorem to be used in $\$ 4$.

THEOREM 3.3. If for the points $b_{1}, b_{2}, b_{3}, \cdots$ we have $b_{i+1}-b_{i} \geqq b>0$, $i=1,2,3, \cdots$, then every solution $u_{1}, u_{2}, u_{2} \cdots$ of the equations

$$
\sum b_{i}^{n} u_{i}=\mu_{n}, \quad n=1,2,3, \cdots,
$$

has absolutely convergent sums in the left member.

Suppose for some integer $m$

$$
\sum_{i=1}^{\infty} b_{i}^{m}\left|u_{i}\right|=\infty
$$

Then for an infinite sequence of indices $l_{2}<l_{8}<l_{4}<\cdots$,

$$
\begin{aligned}
b_{l_{i}}^{m}\left|u_{l_{i}}\right|>\frac{1}{i(\log i)^{n / 2}}, & i=3,4, \cdots, \\
b_{l_{i}}^{m+2}\left|u_{l_{i}}\right|>\frac{l_{i}^{2} b^{2}}{i(\log i)^{3 / 2}}, & i=3,4, \cdots, \\
b_{l_{i}}^{m+2}\left|u_{l_{i}}\right|>b^{2}, & i=3,4, \cdots .
\end{aligned}
$$


Hence the sums diverge for $n \geqq m+2$, contradicting the hypothesis.

For the example $u_{i}=(-1)^{i} / i(\log i)^{3 / 2}, b_{i}=\log i$, the sums converge conditionally, although $\sum_{i=1}^{\infty}\left|u_{i}\right|$ converges.

4. An example. For $(\mu, 0), \mu_{0}=0$, and for arbitrary $\epsilon>0, a \geqq 2$, we here construct a step function solution $\phi(t)$ whose discontinuities are all at the points $a, a^{2}, a^{3}, \cdots$, and whose total variation is less than $\epsilon$.

Consider, for any fixed integer $r$, the infinite system

$$
\begin{aligned}
& \delta_{r 0}=u_{1} \\
& 0=u_{2}+u_{3} \\
& \delta_{r 1}=u_{1}+a u_{2}+a^{2} u_{3} \\
& 0=u_{4}+u_{5}+u_{0} \\
& 0=\quad u_{4}+a u_{6}+a^{2} u_{6} \\
& \delta_{r 2}=u_{1}+a^{2} u_{2}+a^{4} u_{8}+a^{6} u_{4}+a^{8} u_{5}+a^{10} u_{6}
\end{aligned}
$$

where, if $\sigma_{k}=k(k-1) / 2$, the $\left(\sigma_{k+1}\right)$ th equation is

$$
\delta_{r, k-1}=\sum_{i=1}^{\sigma k+1} a^{(i-1)(k-1)} u_{i} \equiv P_{r, k-1}, \quad k=1,2,3, \cdots,
$$

and the $\left(\sigma_{k+1}+l\right)$ th equation is

$$
0=\sum_{i=1}^{k+1} a^{(i-1)(l-1)} u_{\sigma_{k+1}+i} \equiv Q_{r k l}, \quad l=1,2, \cdots, k .
$$

TheOREM 4.1. For any integer $r$ and any $a \neq 1$ the system (4.1) has $a$ unique solution $u_{1}, u_{2}, u_{3}, \cdots$. For $|a|>1$ this solution satisfies the system

$$
\sum_{n=1}^{\infty} a^{(n-1) h} u_{n}=\delta_{r h}, \quad h=0,1,2, \cdots,
$$

the sums converging absolutely.

Let the determinant $A_{n k} \equiv\left|\alpha_{i j}\right|, k \geqq n-1$, where $\alpha_{i j}=a^{(i-1)(j-1)}$, $i=1,2, \cdots, n-1$, and $\alpha_{i n}=a^{k(n-1)}$. Let $A_{n k}^{(l)}$ be the cofactor of $a^{k(l-1)}$ in $A_{n k}$. It is readily verified that

$$
\frac{A_{n, n-1}^{(l)}}{A_{n, n-1}}=\frac{(-1)^{n+l}}{a^{\sigma l-1+(l-1)(n-l)}(a-1) \cdots\left(a^{l-1}-1\right)(a-1) \cdots\left(a^{n-l}-1\right)},
$$




$$
\frac{A_{n k}}{A_{n, n-1}}=\frac{\left(a^{k-n+2}-1\right) \cdots\left(a^{k}-1\right)}{(a-1) \cdots\left(a^{n-1}-1\right)} .
$$

For any $r$ define $S_{j}=\delta_{r, j-1}, j \leqq r+1$, and $S_{j}=-\sum_{1}^{\sigma_{j}} a^{(i-1)(j-1)} u_{i}$ for $j>r+1$. Applying the above determinant formulas to system (4.1) we have for $j=1,2,3, \cdots$, and $i=1,2, \cdots, j$,

$$
\begin{aligned}
& u_{\sigma_{j}+i}=\frac{(-1)^{i+j} S_{j}}{a^{\sigma_{j}(j-1)+(j-i)(i-1)+\sigma_{i-1}}(a-1) \cdots\left(a^{i-1}-1\right)(a-1) \cdots\left(a^{j-i}-1\right)} \\
& S_{j}=-\sum_{l=r+1}^{j-1} a^{\sigma l(j-l)} \frac{\left(a^{j-l+1}-1\right) \cdots\left(a^{j-1}-1\right)}{(a-1) \cdots\left(a^{l-1}-1\right)} S_{l}
\end{aligned}
$$

These equations give the solution of the system (4.1) for $a \neq 1$.

Using the fact that the fraction in the coefficient of $S_{l}$ in (4.6) reduces to a polynomial of degree $(j-l)(l-1)$ with the sum of its coefficients $C_{j-1, l-1}$, we can establish the inequality

$$
\left|S_{j}\right|<|a|^{\sigma_{j-1}(j+3) / 3}[(j-1) !]^{3 / 2}, \quad j \geqq 4,|a|>1 .
$$

This, with (4.5), shows that the sums in (4.4) converge absolutely for $|a|>1$. We have, for any $r$, using (4.2) and (4.3),

$$
\begin{aligned}
\sum_{n=1}^{\infty} a^{(n-1) h} u_{n} & =P_{r h}+\sum_{i=2}^{\infty} a^{\sigma h+i h} Q_{r, h+i-1, h+1} \\
& =\delta_{r h},
\end{aligned}
$$

since a subsequence of the partial sums converges to $\delta_{r h}$. This completes the proof of the theorem.

In constructing the function $\phi(t)$ we observe, using (4.5) and (4.7), that for $a \geqq 2,\left|u_{n}\right|<2^{-n}, n \geqq 7$, so that $\sum\left|u_{n}\right|=\sum_{1}^{0}\left|u_{n}\right|+\sum_{7}^{\infty}\left|u_{n}\right|$ $<9 / 8$ for $r \geqq 1$. Put

$$
\psi_{r}(t)= \begin{cases}0, & 0 \leqq t<a, \\ \sum_{i=1}^{k} u_{i}, & a^{k-1} \leqq t<a^{k}, k=1,2,3, \cdots,\end{cases}
$$

and then $\int_{0}^{\infty} t^{n} d \psi_{r}(t)=\delta_{n r}, n=1,2,3, \cdots$, and $\int_{0}^{\infty}\left|d \psi_{r}(t)\right|<9 / 8$.

Now for arbitrary $\mu_{1}, \mu_{2}, \mu_{3}, \cdots, a \geqq 2, \epsilon>0$, and for each index $r=1,2,3, \cdots$, let $l_{r}$ be the smallest positive integer for which $a^{r l_{r}}>(9 / 8) \cdot 2^{r}\left|\mu_{r}\right| \epsilon^{-1}$, and for $\phi_{r}(t)=\mu_{r} a^{-r l} \psi_{r}\left(a^{-l_{r} t}\right)$ we have

$$
\int_{0}^{\infty} t^{n} d \phi_{r}(t)=\delta_{r n} \mu_{r}, \quad n, r=1,2,3, \cdots,
$$

and 


$$
\int_{0}^{\infty}\left|d \phi_{r}(t)\right|<2^{-r} \epsilon_{.}
$$

Then $\phi(t)=\sum_{1}^{\infty} \phi_{r}(t)$ satisfies

$$
\int_{0}^{\infty} t^{n} d \phi(t)=\mu_{n}, \quad n=1,2,3, \cdots,
$$

and

$$
\int_{0}^{\infty}|d \phi(t)|<\epsilon
$$

Moreover the points of change of $\phi(t)$ are all included among the points $a, a^{2}, a^{3}, \cdots$, and by Theorem 3.3 the integrals converge absolutely.

\section{REFERENCES}

1. R. P. Boas, The Stieltjes moment problem for functions of bounded variation, Bull. Amer. Math. Soc. vol. 45 (1939) pp. 399-404.

2. G. Polya, Sur l'indetermination d'un theorème voisin du problème des moments, C. R. Acad. Sci. Paris vol. 207 (1938) pp. 708-711.

3. - Eine einfache, mit functionen theoretischen Aufgaben verknipfte, hinreichende Bedingung für die Auflösbarkeit eines Systems unendlich vieler linearer Gleichungen, Comment. Math. Helv. vol. 11 (1938) pp. 234-252.

Miami University AND

NORTHWESTERN UNIVERSITY 